\title{
Napping and the risk of type 2 diabetes: a population-based prospective study
}

\section{Hublin, Chister}

2016-01

Hublin , C , Lehtovirta , M , Partinen , M , Koskenvuo , M \& Kaprio , J 2016 , ' Napping and the risk of type 2 diabetes: a population-based prospective study ' , Sleep Medicine , vol. 17 , pp. 144-148 . https://doi.org/10.1016/j.sleep.2015.11.004

http://hdl.handle.net/10138/223867

https://doi.org/10.1016/j.sleep.2015.11.004

publishedVersion

Downloaded from Helda, University of Helsinki institutional repository.

This is an electronic reprint of the original article.

This reprint may differ from the original in pagination and typographic detail.

Please cite the original version. 
Original Article

\title{
Napping and the risk of type 2 diabetes: a population-based prospective study
}

\author{
Christer Hublin ${ }^{\mathrm{a}, \mathrm{b}, *}$, Mikko Lehtovirta ${ }^{\mathrm{c}}$, Markku Partinen ${ }^{\mathrm{b}, \mathrm{d}}$, Markku Koskenvuo ${ }^{\mathrm{b}}$, \\ Jaakko Kaprio b,c,e \\ a Finnish Institute of Occupational Health, Helsinki, Finland \\ ${ }^{\mathrm{b}}$ Department of Public Health, University of Helsinki, Helsinki, Finland \\ c Institute for Molecular Medicine Finland, University of Helsinki, Helsinki, Finland \\ ${ }^{\mathrm{d}}$ Helsinki Sleep Clinic, Vitalmed Research Centre, Helsinki, Finland \\ e National Institute for Health and Welfare, Helsinki, Finland
}

\section{A R T I C L E I N F O}

\section{Article history:}

Received 6 August 2015

Received in revised form 27 October 2015

Accepted 2 November 2015

Available online 2 December 2015

\section{Keywords:}

Type 2 diabetes

Napping

Population

Follow-up

\begin{abstract}
A B S T R A C T
Objective: Some studies indicate an association between napping and increased risk of type 2 diabetes. We studied this prospectively in a sample representative of general population.

Methods: A questionnaire was administered to the Finnish Twin Cohort in 1990 (response rate 77\%, age 33-60 years). The study population included 12,244 subjects who replied to the question "Do you sleep during the daytime (take naps)?" with five response alternatives ranging from "no need" to "every or almost every day." Information on incident cases of diabetes was obtained by linkage to nationwide registers. Logistic regression models were used to obtain odds ratios (ORs) (95\% confidence intervals) for incident type 2 diabetes risk in 1991-2004 by napping category. Adjustments were made for 11 sociodemographic and lifestyle covariates. For subjects aged 33-45 years at baseline, a questionnaire in 2011 provided information on prevalent diabetes.

Results: Thirty-four per cent had no need for napping, and $15 \%$ did so on $\geq 3$ days weekly. There were 356 incident type 2 diabetes cases during the follow-up. Using the 'no need' category as the reference, the risk of type 2 diabetes was significantly increased only among those napping most frequently [OR 1.86 (1.29-2.67), age- and sex-adjusted]. After adjusting for other covariates, the results were essentially the same, but when adjusted for body mass index, the association decreased (to about 1.3) and was statistically non-significant. Analysis of 2011 self-reported type 2 diabetes was in line with the register data.

Conclusions: Frequent napping is associated with future risk of type 2 diabetes. This association is largely explained by obesity.
\end{abstract}

(C) 2015 Elsevier B.V. All rights reserved.

\section{Introduction}

A growing number of epidemiological studies have linked sleep disturbances (insufficient sleep, fragmented sleep, circadian dysregulation, and obstructive sleep apnea) with adverse metabolic sequelae, including obesity, insulin resistance, and type 2 diabetes [1]. Also, short sleep (less than 5-6 h per night) and long sleep ( $8 \mathrm{~h}$ or more), as well as insomnia symptoms, are associated with a significantly increased risk of type 2 diabetes [2].

Napping is a common cross-cultural phenomenon occurring across the lifespan and increasing with advancing age [3]. There are, however, no widely accepted definitions or criteria of napping; it

\footnotetext{
* Corresponding author. Finnish Institute of Occupational Health, Topeliuksenkatu 41 a A, Helsinki FIN-00250, Finland. Tel.: +358 30474 2120; fax: +358 92412414.

E-mail address: christer.hublin@ttl.fi (C. Hublin).
}

usually refers to brief periods of daytime sleep (lasting less than several hours). Naps have also been used as a countermeasure to sleepiness during night shifts at work. In a review of earlier napping literature, Dinges [4] concluded that three-quarters of adults reported napping and the usual duration was from about 30 to $90 \mathrm{~min}$. Later, Pilcher et al. [5] showed in a one-week sleep-log study in a group of young and middle-aged adults that in almost half of them, the average nap lasted less than 20 min (often called power naps).

Napping has been regarded both as a health-promoting habit [3] and as a risk factor, or a proxy, for a variety of chronic conditions [6] and mortality [7]. Among the most notable of these adversities are obesity and metabolic syndrome [8]. Obesity is both a major risk factor for type 2 diabetes [9] and also increases the risk for sleepdisordered breathing, especially obstructive sleep apnea [10]. Furthermore, while excessive daytime sleepiness and need for naps can result from poor sleep, it has also been shown that a shared 
underlying factor, like chronic stress exposure, both promotes obesity and deteriorates sleep [11].

Contrary to the many studies on other aspects of sleep and metabolic changes, there are only few reports of the association between napping and diabetes [8,12-15], fasting hyperglycemia [16], glycated hemoglobin, or insulin resistance [17], respectively. These available studies suggest an association between napping and diabetes (mainly type 2 diabetes) or impaired glucose regulation. However, the direction of possible causation and the existence of known or unknown confounders have been inadequately explored.

\subsection{Aims}

In this study we assessed the association between napping and the risk of type 2 diabetes in a large, prospective, populationbased cohort of Finnish adult twins, with 14-year follow-up data and detailed information on potential confounding or effectmodifying factors, and information on incident cases of diabetes from nationwide registers. We also examined the association among a subset of twins replying to a follow-up questionnaire. We wanted especially to address the role of obesity in this association.

\section{Methods}

\subsection{Population sample}

The Older Finnish Twin Cohort is a longitudinal study of Finnish twin pairs of the same gender born before 1958 with both cotwins alive in 1975. These pairs were selected from the Central Population Registry of Finland in 1974 [18]. Zygosity was determined using an accurate and validated questionnaire method [19]. The third questionnaire survey in 1990 was mailed to pairs born in 1930-1957 with both co-twins resident in Finland in 1987, and 16,179 twin individuals could be contacted (response rate $77.3 \%$; $N=12,502$; $54.4 \%$ women; mean age in 199043.9 years). The questionnaire included 103 multiple-choice questions, of which 22 were sleep- and vigilance-related. A fourth questionnaire survey was conducted in 2011-2012 among twins born in 1945-1957 [20]. This subpopulation included 5419 subjects (450 type 2 diabetes cases) who replied in both 1990 and 2011.

The ethical committee of the Department of Public Health, University of Helsinki has approved the study. Informed consent was obtained from all respondents.

\subsection{Questionnaire and register data}

The study population included 12,244 subjects who replied to the question "Do you sleep during the daytime (take naps)?" with response alternatives: (1) no need; (2) I would like to but I cannot sleep during the daytime; (3) on two days weekly or more seldom; (4) on three to five days weekly; or (5) every or almost every day. Additionally there were questions on working (working outside home / housekeeper, working at home / retired / studying / unemployed); working time (regular daytime work / regular nighttime work / shiftwork without nightshift / shiftwork with nightshift / not working) [21]; height in $\mathrm{cm}$ and weight in $\mathrm{kg}$ to calculate BMI as (weight in $\mathrm{kg}$ )/(height in meters) ${ }^{2}$; use of alcohol (more than five bottles of beer or comparable amount at least once monthly yes/ no, number of pass outs during the last year) [22,23]; smoking (never / occasional / former / current); sleep length (6 h or less / 6.5 / 7 | 7.5 / 8 / 8.5 / 9 / 9.5 / 10 h or more) [24]; and excessive sleepiness in the morning or during the day [25]; snoring; and insomnia (all asked using the following alternatives: every or almost every day / three to five days weekly / one to two days weekly / less than weekly / less than monthly or never) [21]. Information on diurnal type was from the 1981 questionnaire, using the question, "Will you try to estimate to what extent you are a morning or an evening person" with reply alternatives, "I am clearly a morning person", "I am to some extent a morning person", "I am to some extent an evening person", and “I am clearly an evening person” [26]. The 2011 questionnaire included a self-report of type 2 diabetes.

Information on incident cases of diabetes in 1991-2004 was obtained by linkage of nationwide registers, using the unique personal ID assigned to all Finns, from death certificates, the National Hospital Discharge Register and the Medication Register of the Social Insurance Institution, and classified as type 1 diabetes, type 2 diabetes, and other as described in detail elsewhere [9,27]. Those having diabetes onset prior to 1991 were excluded based on 1975 and 1981 questionnaire data and on follow-up from nationwide registers during 1975-1991. Data on vital status during follow-up were obtained from the Population Register Centre of Finland, which holds information on deaths, migration, or current address in Finland. In the fourth questionnaire survey in 2011, subjects were asked if they had ever been diagnosed with diabetes by a physician. Response alternatives were: never; no but elevated blood sugar; gestational diabetes; type 1 diabetes; type 2 diabetes; could not say which type of diabetes. We examined risk of type 2 diabetes vs never in relation to napping.

\subsection{Data analysis and statistical methods}

Standard statistical methods were used in data analysis and implemented in the Stata package (version 13.1, Stata Corp, College Station, TX, USA, www.stata.com). Logistic regression models were used to obtain odds ratios (ORs) and their 95\% confidence intervals $(\mathrm{CI})$ for risk of incident type 2 diabetes by napping class (no need; I would like to but I cannot sleep during the daytime; on two days weekly or more seldom; on three to five days weekly; or every or almost every day). Those having diabetes onset prior to 1991 were excluded. Logistic regression was used instead of survival models as type 2 diabetes typically has an insidious onset and thus a firm date of diagnosis cannot be defined unambiguously.

We selected potential covariates that are known or suspected risk factors for type 2 diabetes and have been associated with sleep behaviors, including napping. In addition they had to be available in the data. We tested for the equality of means of continuous variables by napping categories using a one-way analysis of variance approach, while associations of napping categories with categorical variables was done using chi-squared tests. Logistic regression models were used for estimating the odds ratios of type 2 diabetes by napping categories. Covariates were added in a stepwise procedure to illustrate their effect, resulting in four models.

Means and frequency distributions of the variables did not differ by zygosity, indicating that the twin individuals come from the same base population. This was an analysis of twins as individuals; as they had been sampled as twin pairs, the statistical lack of independence of two observations in a pair was taken into account by using complex survey methods with twin pair membership as the primary sampling unit in analyses (Stata svy procedures) or using a robust estimator of variance to derive proper standard errors and hence $p$-values (cluster option in Stata). Effectively the modest correlations of twins within pairs for napping reduce the effective sample size and thus $p$-values are consequently larger than if the statistical non-independence is (incorrectly) ignored. However, point estimates (such as odds ratios) are unaffected by these procedures.

\section{Results}

The distribution of answers to the question on napping was as follows: no need, 34.3\%; I would like to but I cannot sleep during the daytime, $15.7 \%$; on two days weekly or more seldom, $35.2 \%$; on three to five days weekly, 7.7\%; and every or almost every day, $7.1 \%$. 
Table 1

Selected demographic characteristics in 1990 of the entire cohort $(N=12,351)$ by response categories to napping.

\begin{tabular}{|c|c|c|c|c|c|}
\hline & No need & $\begin{array}{l}\text { I would like to but I cannot } \\
\text { sleep during daytime }\end{array}$ & $\begin{array}{l}\leq 2 \text { days } \\
\text { weekly }\end{array}$ & $\begin{array}{l}3-5 \text { days } \\
\text { weekly }\end{array}$ & $\begin{array}{l}\text { Every or almost } \\
\text { every day }\end{array}$ \\
\hline Mean age (years $\pm s d$ ) & $43.6 \pm 7.3$ & $44.2 \pm 7.8$ & $43.9 \pm 7.6$ & $46.1 \pm 8.1$ & $48.4 \pm 8.2$ \\
\hline Women $(\%)$ & 51.5 & 65.7 & 54.8 & 48.3 & 49.8 \\
\hline Working outside home (\%) & 79.0 & 71.7 & 78.1 & 71.1 & 57.2 \\
\hline Nighttime work (\%) & 5.5 & 7.2 & 10.9 & 14.2 & 13.2 \\
\hline Obese (BMI $\geq 30 \mathrm{~kg} / \mathrm{m}^{2}$ ) & 6.2 & 7.6 & 8.1 & 9.1 & 14.2 \\
\hline Heavy alcohol use (\%) & 26.7 & 26.0 & 27.2 & 31.8 & 26.4 \\
\hline Current smokers (\%) & 22.4 & 24.2 & 28.4 & 28.6 & 31.3 \\
\hline Mean sleep length $(\mathrm{h})$ & 7.48 & 7.28 & 7.51 & 7.53 & 7.66 \\
\hline Diurnal type: clear eveningness (asked in 1981; \%) & 7.8 & 10.9 & 9.9 & 11.4 & 9.7 \\
\hline Excessive morning sleepiness $\geq 3$ days weekly (\%) & 8.5 & 32.6 & 15.3 & 20.6 & 26.9 \\
\hline Excessive daytime sleepiness $\geq 3$ days weekly (\%) & 3.7 & 27.5 & 11.8 & 32.4 & 48.0 \\
\hline Habitual snoring (\%) & 19.6 & 22.2 & 23.0 & 31.7 & 35.3 \\
\hline Insomnia $\geq 3$ days weekly (\%) & 3.6 & 15.6 & 5.5 & 7.9 & 12.5 \\
\hline
\end{tabular}

The means or frequencies of all covariates differed significantly by napping category.

There were no major differences in the distributions between age groups under $40,40-50$, and 50 years or older, respectively. There were no significant differences between the two genders. Details on these as well as distributions of demographic characteristics and lifestyle covariates by response categories to the question on napping are given in Table 1.

There were 356 incident type 2 diabetes cases during the 14year follow-up. Using the 'no need' category as the reference, the risk of type 2 diabetes was significantly increased only among those napping most frequently [1.86 (1.29-2.67), age- and sex-adjusted]. The ORs for the other reply alternatives were as follows: I would like to but I cannot sleep during the daytime, 1.14 (0.81-1.61); on two days weekly or more seldom, 1.25 (0.95-1.63); and on three to five days weekly, 0.97 (0.63-1.51).

The risk for type 2 diabetes remained essentially unchanged [OR for most frequent nappers $=1.56(1.04-2.34)$ ] after adjusting (in addition to age and sex) for work, working time, use of alcohol, sleep length, sleepiness in the morning, insomnia, or snoring. However, after additionally adjusting for sleepiness during the day [1.25 (0.791.97)] or BMI [1.37 (0.89-2.12)], the risk estimate for the most frequent napping category became non-significant (Table 2). Adjustment for smoking status or diurnal type had no major effect on the results. We did not conduct any pairwise analysis to study within pair associations of napping with type 2 diabetes incidence given that the association on the individual level was no longer significant after adjustment for key covariates.

When adjusted for sex and age, the self-reported diabetes risk in 2011 questionnaire was significant [1.71 (1.12-2.61)] for those napping daily or almost daily, but the risk decreased and became non-significant [1.42 (0.89-2.29)] when additionally adjusted for BMI.

\section{Discussion}

Our results show that the risk for type 2 diabetes was significantly increased among middle-aged individuals who, as much as 14 years earlier, had reported napping daily or almost daily. The association remained essentially the same after adjustments for sleeprelated covariates such as insomnia, snoring, or sleep length. However, the association became non-significant after adjusting for BMI, suggesting that napping is not an independent risk factor for type 2 diabetes. This conclusion was further supported by the similar results of the self-reported diabetes risk in the 2011 questionnaire.

There are a few previous studies assessing the association between napping and diabetes or impaired glucose regulation. In a US sample $(N=235)$ of older individuals (mean age 80 years), napping was significantly increased ( $O R=6.1$, adjusted for $B M I)$ among those having self-reported diabetes [12]. In another US study including 414 community-dwelling older (70-89 years) adults, napping was similarly increased among self-reported diabetics $(\mathrm{OR}=1.87$, adjusted for BMI) [13]. In a third US study focusing on ethnic-specific differences in napping duration in postmenopausal women with type 2 diabetes, a significant association was found between long nap duration ( $\geq 30 \mathrm{~min} /$ day) and diabetes among White women [15].

In the Quangzhou Biobank Cohort Study [8] 19,567 participants (mean age above 60 years) were selected from a community social and welfare association, and type 2 diabetes was assessed by fasting blood glucose $(\geq 7.0 \mathrm{mmol} / \mathrm{L})$ and/or self-reports of physician diagnosis or treatment. The risk of type 2 diabetes was significantly increased (ORs around 1.3 ) in those napping on $\geq 4$ days weekly (adjusted for waist circumference). Another Chinese study based on a cohort $(N=27,009)$ of retired workers (mean age 63.6

Table 2

Napping and risk of incident type 2 diabetes (odds ratio and 95\% confidence interval) in 1991-2004.

\begin{tabular}{|c|c|c|c|c|}
\hline Napping category & Model $1^{\mathrm{a}}$ & Model $2^{\mathrm{b}}$ & Model $3^{c}$ & Model $4^{\mathrm{d}}$ \\
\hline No need (reference) & 1.00 & 1.00 & 1.00 & 1.00 \\
\hline I would like to but cannot sleep during the daytime & $1.14(0.81,1.61)$ & $1.15(0.80,1.67)$ & $1.06(0.73,1.55)$ & $1.13(0.76,1.67)$ \\
\hline Two days weekly or more seldom & $1.25(0.95,1.63)$ & $1.30(0.98,1.72)$ & $1.23(0.92,1.65)$ & $1.25(0.93,1.68)$ \\
\hline Three to five days weekly & $0.97(0.63,1.51)$ & $1.04(0.67,1.60)$ & $0.91(0.58,1.42)$ & $1.01(0.64,1.60)$ \\
\hline Every or almost every day & $1.86(1.29,2.67)$ & $1.56(1.04,2.34)$ & $1.25(0.79,1.97)$ & $1.37(0.89,2.12)$ \\
\hline
\end{tabular}

a Model 1 is adjusted for: age and sex.

b Model 2 is Model 1 additionally adjusted for: work, working time, use of alcohol, sleep length, sleepiness in the morning, insomnia, and snoring.

c Model 3 is Model 2 additionally adjusted for: sleepiness during the day.

d Model 4 is Model 2 additionally adjusted for: BMI. 
years) using laboratory tests and not differentiating diabetes type (but stating that more than 95\% of all older diabetics in China have type 2 diabetes) found significantly increased risk of diabetes (ORs 1.14 ) in nap duration from 30 to less than $60 \mathrm{~min}$ and in $\geq 90 \mathrm{~min}$ (but not in the duration category between; adjusted for BMI) [16]. A third Chinese study including 7568 non-diabetic participants (mean age 51 years) found a significant increase in glycated hemoglobin (HbA1c > 6.0\%; ORs around 1.3) and in insulin resistance among daily nappers (ORs around 1.6, all adjusted for BMI) [17]. In addition to these previously mentioned cross-sectional studies, there is one prospective US study on daily napping (asked in 1996-1997) and risk of self-reported diabetes (diagnosed between 2000 and 2006; not differentiating the type but stating that about $90-95 \%$ is type $2 \mathrm{di}-$ abetes) in adults aged 50 years or more $(N=174,542)$ participating in the NIH-AARP Diet and Health Study [14]. Compared to no napping, a significantly increased risk of diabetes was found among those napping $<1 \mathrm{~h}(\mathrm{OR}=1.16)$ and $\geq 1 \mathrm{~h}(\mathrm{OR}=1.37$, both adjusted for BMI). In all, these previous results suggest an association between napping and diabetes or impaired glucose regulation.

Methodological aspects and differences may at least partly explain why our results are not fully in line with the earlier studies. First, all previous studies apart from Xu et al. [14] are cross-sectional and therefore it is difficult to assess whether napping could be a causative factor or a response to the change in glucose metabolism. Second, in many of the previous studies, the diabetes-diagnosis was solely [12-14] or partly [8] based on self-report and they did not differentiate between type 1 and type 2 diabetes, although it is obvious - based on the age-groups studied - that the vast majority had type 2 diabetes. In three studies [8,16,17] diabetes or milder impairment in glucose metabolism was identified with one-time laboratory tests. Our study is the first based on a nationwide register of diagnosed type 2 diabetes patients. Third, in the other prospective study by $\mathrm{Xu}$ et al. [14], the self-report of diabetes was asked 3-10 years after the questioning of the napping pattern and it is possible that at least among those with short follow-up there may have been a considerable proportion of cases with undiagnosed diabetes at the time-point when asking about napping. Fourth, the duration of our study enabled more incident cases of diabetes to be detected: more than two-thirds of the cases were identified during the second half of the 14-year follow-up time (data not shown).

Our study has also weaknesses. We did not have systematic information about waist circumferences. As a marker of visceral fat, waist circumference is an important indicator of insulin resistance and type 2 diabetes [27-29]. Although BMI and waist circumference correlate with each other, the latter seems to be more strongly associated with type 2 diabetes than BMI [30,31]. We cannot, thus, explain the degree to which the association between napping and type 2 diabetes is accounted for by general and on the other hand by visceral adiposity. BMI was not based on measured weight and height, but as the correlation between measured and questionnaire-based BMI has shown to be 0.9 in this cohort [32], we conclude that the method used in the present study is reliable.

Studies on napping and health have also been inconclusive [33]. A short daytime 10- to 45-min sleep has been shown to improve mood and performance in a variety of settings [6]. On the other hand, longer and frequent naps have been associated with increased morbidity and mortality among older [34], and in some studies also among younger [35] individuals. Adverse effects associate with excess daytime sleepiness and deterioration of several circadian patterns. In line with this, in our study the risk for type 2 diabetes was increased among subjects who took a nap frequently, were overweight, and reported excess daytime sleepiness. In fact, we already have shown with the same study cohort that BMI estimates recorded already 15 years before the napping questionnaire used here almost unequivocally predicted the risk for type 2 diabetes [9]. As compared to normal-weight subjects, the risk for type 2 diabetes among obese subjects was 6.8-fold, and 13.64-fold among morbidly obese subjects, respectively [9]. Although we do not have older napping records, we cannot exclude the possibility that obesity preceded napping in our study.

In conclusion, epidemiological studies, the current study included, have not been able to provide solid evidence for an independent role of napping in type 2 diabetes occurrence. A more plausible explanation seems to be that napping becomes more common with increasing obesity and the associated increase in disturbed metabolism.

\section{Conflict of interest}

The authors report no conflicts of interest.

The ICMJE Uniform Disclosure Form for Potential Conflicts of Interest associated with this article can be viewed by clicking on the following link: http://dx.doi.org/10.1016/j.sleep.2015.11.004.

\section{Acknowledgement}

This research was supported by the Academy of Finland (Grant nos. 263278, 265240, and 267882).

\section{References}

[1] Koren D, O’Sullivan KL, Mokhlesi B. Metabolic and glycemic sequelae of sleep disturbances in children and adults. Curr Diab Rep 2015;15:562-71. doi:10.1007/s11892-014-0562-5.

[2] Cappuccio FP, D'Elia L, Strazzullo P, et al. Quantity and quality of sleep and incidence of type 2 diabetes: a systematic review and meta-analysis. Diabetes Care 2010;33:414-20.

[3] Milner CE, Cote KA. Benefits of napping in healthy adults: impact of nap length, time of day, age, and experience with napping. J Sleep Res 2009;18:272-81.

[4] Dinges DF. Napping patterns and effects in human adults. In: Dinges DF, Broughton RJ, editors. Sleep and alertness: chronobiological, behavioral, and medical aspects of napping. New York: Raven Press; 1989. p. 171-204.

[5] Pilcher JJ, Michalowski KR, Carrigan RD. The prevalence of daytime napping and its relationship to nighttime sleep. Behav Med 2001;27:71-6.

[6] Dhand R, Sohal H. Good sleep, bad sleep! The role of daytime naps in healthy adults. Curr Opin Pulm Med 2006;12:379-82.

[7] Leng Y, Wainwright NW, Cappuccio FP, et al. Daytime napping and the risk of all-cause and cause-specific mortality: a 13-year follow-up of a British population. Am J Epidemiol 2014;179:1115-24.

[8] Lam K-BH, Jiang CQ Thomas GN, et al. Napping is associated with increased risk of type 2 diabetes: the Guangzhou Biobank Cohort Study. Sleep 2010;33:402-7

[9] Lehtovirta M, Pietiläinen KH, Levälahti E, et al. Evidence that BMI and type 2 diabetes share only a minor fraction of genetic variance: a follow-up study of 23,585 monozygotic and dizygotic twins from the Finnish Twin Cohort Study. Diabetologia 2010;53:1314-21.

[10] Wolk R, Shamsuzzaman ASM, Somers VK. Obesity, sleep apnea, and hypertension. Hypertension 2003;42:1067-74.

[11] Lucassen EA, Cizza G. The hypothalamic-pituitary-adrenal axis, obesity, and chronic stress exposure: sleep and the HPA axis in obesity. Curr Obes Rep 2012;1:208-15.

[12] Goldman SE, Hall M, Boudreau R, et al. Association between nighttime sleep and napping in older adults. Sleep 2008;31:733-40.

[13] Picarsic JL, Glynn NW, Taylor CA, et al. Self-reported napping and duration and quality of sleep in the lifestyle interventions and independence for elders pilot study. J Am Geriatr Soc 2008;56:1674-80.

[14] Xu Q, Song Y, Hollenbeck A, et al. Day napping and short night sleeping are associated with higher risk of diabetes in older adults. Diabetes Care 2010;33:78-83.

[15] Shadyab AH, Kritz-Silverstein D, Laughlin GA, et al. Ethnic-specific associations of sleep duration and daytime napping with prevalent type 2 diabetes in postmenopausal women. Sleep Med 2015;16:243-9.

[16] Fang W1, Li Z, Wu L, et al. Longer habitual afternoon napping is associated with a higher risk for impaired fasting plasma glucose and diabetes mellitus in older adults: results from the Dongfeng-Tongji cohort of retired workers. Sleep Med 2013;14:950-4.

[17] Baoying H, Hongjie C, Changsheng Q et al. Association of napping and nighttime sleep with impaired glucose regulation, insulin resistance and glycated haemoglobin in Chinese middle-aged adults with no diabetes: a cross-sectional study. BMJ Open 2014;4:e004419. doi:10.1136/bmjopen-2013-004419.

[18] Kaprio J, Koskenvuo M. Genetic and environmental factors in complex diseases: the older Finnish Twin Cohort. Twin Res 2002;5:358-65. 
[19] Sarna S, Kaprio J, Sistonen P, et al. Diagnosis of twin zygosity by mailed questionnaire. Hum Hered 1978;28:241-54.

[20] Kaprio J. The Finnish Twin Cohort Study: an update. Twin Res Hum Genet 2013;16:157-62.

[21] Hublin C, Partinen M, Koskenvuo M, et al. Heritability and mortality risk of insomnia-related symptoms: a genetic epidemiologic study in a populationbased twin cohort. Sleep 2011;34:957-64.

[22] Kaprio J, Koskenvuo M, Langinvainio H, et al. Genetic influences on use and abuse of alcohol: a study of 5638 adult Finnish twin brothers. Alcohol Clin Exp Res 1987; 11:349-56.

[23] Romanov K, Rose RJ, Kaprio J, et al. Self-reported alcohol use: a longitudinal study of 12,994 adults. Alcohol Alcohol Suppl 1987;1:619-23.

[24] Hublin C, Partinen M, Koskenvuo M, et al. Sleep and mortality: a populationbased 22-year follow-up study. Sleep 2007;30:1245-53.

[25] Hublin C, Kaprio J, Partinen M, et al. Daytime sleepiness in an adult, Finnish population. J Intern Med 1996;239:417-23.

[26] Koskenvuo M, Hublin C, Partinen M, et al. Heritability of diurnal type: a nationwide study of 8753 adult twin pairs. J Sleep Res 2007;16:156-62.

[27] Kaprio J, Tuomilehto J, Koskenvuo M, et al. Concordance for type 1 (insulindependent) and type 2 (non-insulin-dependent) diabetes mellitus in a population-based cohort of twins in Finland. Diabetologia 1992;35: 1060-7.
[28] Feng RN, Zhao C, Wang C, et al. BMI is strongly associated with hypertension, and waist circumference is strongly associated with type 2 diabetes and dyslipidemia, in northern Chinese adults. J Epidemiol 2012;22:317-23.

[29] Mullington JM, Hinze-Selch D, Pollmacher T. Mediators of inflammation and their interaction with sleep: relevance for chronic fatigue syndrome and related conditions. Ann N Y Acad Sci 2001;933:201-10.

[30] Patel SR, Zhu X, Storfer-Isser A, et al. Sleep duration and biomarkers of inflammation. Sleep 2009;32:200-4.

[31] Nazare JA, Smith J, Borel AL, et al. Usefulness of measuring both body mass index and waist circumference for the estimation of visceral adiposity and related cardiometabolic risk profile (from the INSPIRE ME IAA Study). Am J Cardiol 2015;115:307-15.

[32] Korkeila M, Kaprio J, Rissanen A, et al. Predictors of major weight gain in adult Finns: stress, life satisfaction and personality traits. Int J Obes Relat Metab Disord 1998;22:949-57.

[33] Lombardi C, Mattaliano P, Parati G. Is daytime siesta a harmful habit? J Hypertens 2014;32:1959-61.

[34] Hays JC, Blazer DG, Foley DJ. Risk of napping: excessive daytime sleepiness and mortality in an older community population. J Am Geriatr Soc 1996:44:693-8.

[35] Campos H, Siles X. Siesta and the risk of coronary heart disease: results from a population-based, case-control study in Costa Rica. Int J Epidemiol 2000;29:429-37. 